\title{
Desenvolvimento e validação de um modelo preditor do desempenho de novilhos de corte sob suplementação em pastagens tropicais
}

\author{
Diego Avilio Ocampos Olmedo, Jorge López ${ }^{1}$, Júlio Otávio Jardim Barcellos ${ }^{1}$, Tamara \\ Esteves de Oliveira ${ }^{1}$
}

\begin{abstract}
${ }^{1}$ Universidade Federal de Rio Grande do Sul - Departamento de Zootecnia, Av. Bento Gonçalves 7712, CEP: 91540-000, Porto Alegre, RS.
RESUMO - Um modelo de simulação foi desenvolvido e validado com o objetivo de conhecer e predizer o desempenho produtivo de bovinos machos castrados com diferentes pesos e idades iniciais mantidos sobre pastagens cultivadas Panicum sp. e Brachiaria sp. em regiões tropicais e subtropicais. O modelo incluiu relações diretas entre os dois componentes principais (animal e forragem) e relações indiretas entre outros dois componentes (clima e solo). O componente animal incluiu: consumo de matéria seca de forragem, consumo de suplemento, carga animal, peso e idade inicial dos animais, biótipo (peso adulto e peso ao nascimento) e exigências de energia para mantença e produção animal (ganho de peso). O componente forragem incluiu: crescimento, senescência, digestibilidade e disponibilidade. Os resultados obtidos com o modelo foram comparados a dados experimentais obtidos da literatura em condições semelhantes e indicaram baixo (35\%) erro percentual de estimação. O modelo possibilita avaliar determinadas estratégias nutricionais e, desta forma, auxilia em decisões que dependem do ganho de peso dos animais e das condições da pastagem no decorrer do tempo.
\end{abstract}

Palavras-chave: consumo, desempenho, pastejo, simulação

\section{Development and validation of a performance predictive model of beef steers supplemented in tropical pastures}

\begin{abstract}
A simulation model was developed and validated with the objective of evaluating and predicting the productive performance of steers with different weights and initial ages kept on Panicum sp. or Brachiaria sp. cultivated pastures in tropical and subtropical regions. The model established the direct relationships between two main components (animal and fodder plant) and two indirect components (climate and soil). The animal component included: intake of forage dry matter, supplement intake, stocking rate, initial weight and age of the animals, biotype (adult weight and weight at birth), energy requirement for maintenance and production (live weight gain). The forage components included: growth, senescence, digestibility and availability. The results obtained with the model were compared to those obtained in the literature under similar conditions in Brazil and a low percentage of estimated error was found (35\%). The model is an adequate tool to evaluate certain nutritional strategies and thus to assist in decisions that depend on the weight gain of the animals and pasture conditions over time.
\end{abstract}

Key Words: intake, grazing, performance, simulation

\section{Introdução}

Um problema metodológico nos sistemas pecuários em pastejo é a avaliação do real potencial de produção, uma vez que diversos fatores influenciam a produtividade animal, dificultando a experimentação convencional. Assim, os modelos de simulação têm sido utilizados para abordar o problema e formular hipóteses de manejo adequadas.

A integração dos conhecimentos utilizados na produção de carne ocorre por meio da pesquisa em sistemas de produção, cuja estratégia nas formas de produção e manejo é aumentar o uso dos recursos disponíveis. Além disso, ainda é possível definir o sistema pela sua análise utilizando modelos matemáticos. Assim, o pesquisador constrói o modelo que representará um sistema real para predizer seu funcionamento futuro (Allende, 2003).

Existem modelos matemáticos bem definidos das respostas do sistema de produção de animais em confinamento, como os modelos Institut National de la Recherche Agronomique - INRA (1989), Australiam Commonwealth Scientific and Industrial Research Organization - CSIRO (1990), Agricultural and Food Research Council - AFRC (1993) e National Research Council - NRC (2000). Entretanto, esses modelos foram 
desenvolvidos para condições diferentes das encontradas no trópico e subtrópico e em regime de pastejo na América do Sul. Portanto, são limitados à predição de desempenho nos locais de origem, o que poderia ser contornado por meio de novos modelos de simulação locais que disponibilizem maiores recursos para se tomarem decisões, diminuindo as incertezas. Uma boa decisão inclui a avaliação da acurácia, do custo de coleta dos dados e da eficiência com que o sistema pode ser manipulado (Barioni et al., 2002).

Este trabalho foi realizado para desenvolver e validar um modelo de simulação para estimar o ganho de peso de novilhos mantidos em pastejo com ou sem suplementação em pastagens de capim-colonião ou capim-braquiária e avaliar o efeito das variações do valor nutritivo do pasto e do peso dos animais sobre o sistema.

\section{Material e Métodos}

O modelo constitui-se dos modelos animal e de pastagem, divididos em submodelos e avaliados no MSExcel $^{\circledR}$ com macros contendo fórmulas inseridas pela programação Visual Basic. Na construção foi utilizada uma abstração do sistema real para avaliar parâmetros que afetam o sistema em maior escala e para deduzir outras pressuposições de menor magnitude que fazem parte do sistema produtivo simulado (Figura 1).

No modelo animal, o sistema de pastejo influencia a disponibilidade de forragem, a digestibilidade e o consumo dos animais. O modelo assume um pastejo de acordo com o tempo e com a taxa de lotação, em cabeças/ha, fixa ao longo do experimento, determinada pelo usuário. A massa de forragem de $400 \mathrm{~kg} / \mathrm{ha}$ de matéria seca indicou resultados satisfatórios e consistentes.

No submodelo consumo, o consumo de longo prazo em pastejo é o produto cumulativo da ingestão obtida em cada refeição do dia. As características das refeições (duração, número, distribuição ao longo do dia, etc.) e a magnitude do consumo obtido são reflexos diretos da qualidade, quantidade e estrutura do pasto disponível ao animal (Carvalho et al., 2007). Lima et al. (2001) determinaram que o consumo do animal em pastejo oscila entre $2,15 \%$ e $2,37 \%$ do peso corporal. O consumo depende da produção de massa seca da pastagem (Boval et al., 2007), mas, em pastagens tropicais, os efeitos da oferta de forragem associados aos aspectos de altura e estrutura do dossel sobre o consumo de forragem e seu valor nutritivo têm

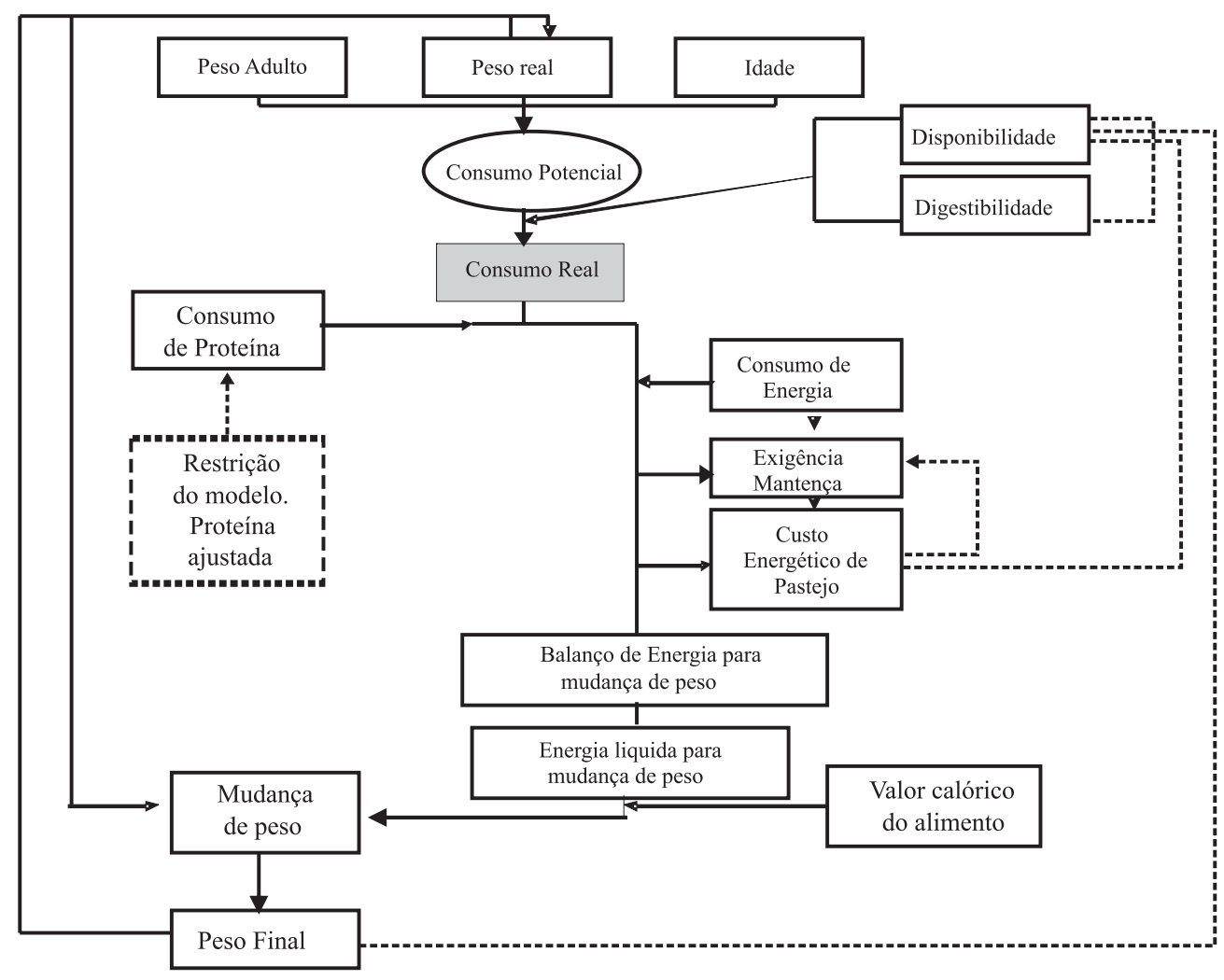

Figura 1 - Modelo da variação do peso dos animais. 
sido pouco avaliados. Alguns estudos, no entanto, comprovam efeito positivo da oferta de forragem sobre a produção de leite ou as taxas de ganho de peso (Gontijo Neto, 2006).

O modelo de consumo potencial (CPO) é baseado nas funções apresentadas no CSIRO (1990), em que:

$$
\begin{aligned}
& \mathrm{N}=\mathrm{A}-(\mathrm{A}-\mathrm{B}) \exp ^{-\mathrm{ke}} \\
& \mathrm{PR}=\mathrm{P} / \mathrm{PA} \\
& \mathrm{CPO}=\mathrm{C} * \mathrm{PA} * \mathrm{PR} *(1,7-\mathrm{PR})
\end{aligned}
$$

Allende (2003) utilizou o fator de digestibilidade para ajustar o consumo potencial e Aguilar (2007) incorporou uma restrição a mais por disponibilidade, denominando-se consumo potencial real, em que:

$$
\mathrm{CPOR}=\mathrm{CPO} * \mathrm{FCDD}(8) * \mathrm{FCDig}(7)
$$

$\mathrm{O}$ conceito de índice de seletividade se baseia no fato de que o animal seleciona de acordo com a diversidade e a disponibilidade de forragem. Supõe-se que, se o animal não tem diversidade, não será capaz de selecionar. O modelo funciona adequadamente com massa seca de forragem superior a $1.000 \mathrm{~kg}$ MS/ha e, nestes casos, o índice de seleção será de 1,0 e a digestibilidade do ingerido, igual à do material oferecido. A diferença entre a digestibilidade consumida e oferecida resulta em valor máximo de $25 \%$. Portanto, é um pressuposto de que a capacidade máxima de seleção pelo animal ocorre quando a digestibilidade é baixa em condições de alta disponibilidade de forragem. Por outro lado, quando a digestibilidade é muito alta ( $80 \%)$, IS $=1,0$.

$$
\begin{aligned}
& \text { IS }=1+\text { SD. SC } \\
& \mathrm{SC}=0,5-0,625 \text { DGO quando } 0,4<\text { DGO }<0,8
\end{aligned}
$$

Assume-se uma seletividade máxima em percentual de utilização da pastagem em torno de $10 \%$ e torna-se difícil para o animal selecionar quando o percentual de utilização se aproxima de $50 \%$, ou seja, com alta disponibilidade de forragem. O índice de seletividade é corrigido na forma linear entre esses dois pontos assumindo-se que a relação entre consumo e digestibilidade da forragem é linear.

$$
\begin{aligned}
& \mathrm{SD}=1,25-2,5 * \mathrm{PU} 0,1<\mathrm{PU}<0,5 \\
& \mathrm{SC}=1 \text { quando } \mathrm{PU}<1 \text { e } \mathrm{SC}=0 \text { quando } \mathrm{PU}=0,5 \\
& \mathrm{DGC}=\mathrm{DGO}+\mathrm{IS}
\end{aligned}
$$

O consumo voluntário aumenta de forma linear, de acordo com a digestibilidade, até o valor máximo de $80 \%$ (Allende, 2003). Neste trabalho é apresentada a função de correção da digestibilidade, com ajuste linear de um fator de correção para a faixa de digestibilidade de 40 a $80 \%$.

$$
\mathrm{FCDig}=1,675 * \mathrm{DGC}-0,34
$$

A relação entre consumo e disponibilidade de forragem é representada por uma função do tipo exponencial assintótico, baseada na fórmula apresentada por Castellaro et al. (2007):

$$
\mathrm{FCD}=1-\operatorname{EXP}(-0,001664 * \mathrm{DD})
$$

As casualizações do consumo são feitas simulando uma distribuição normal com a média no valor calculado e finalmente o consumo voluntário é calculado pelas fórmulas a seguir:

$$
\begin{aligned}
& \mathrm{Zo}=2 * \mathrm{RND}-1 \\
& \mathrm{Z}=\{\log [(1+\mathrm{zo}) /(1-\mathrm{zo})]\} / 1,82 \\
& \mathrm{CVOF}=\mathrm{CPOR} * \mathrm{Z} * 0,07+\mathrm{CPOR}
\end{aligned}
$$

A relação entre consumo de forragem e concentrado baseia-se no grau de substituição, que é aparentemente proporcional à digestibilidade da forrageira. A equação que melhor descreve a relação é a desenvolvida por Holmes \& Jones (1964).

$$
\mathrm{CF}=1,27-0,0154 \mathrm{DD}
$$

Na construção do modelo, foi utilizada a hipótese de que o modelo, diante de uma restrição moderada de forragem, sofresse efeito sinérgico pela adição do concentrado até o momento em que o animal atingisse o nível de consumo potencial (CPO), determinado pela qualidade da forragem. Após o consumo total do concentrado, se o consumo calculado de MS for superior ao consumo calculado de forragem, o excesso é ajustado pela equação anterior para determinar o efeito aditivo líquido. Se o consumo real da forrageira somado ao de concentrado for inferior ao calculado para a forrageira, o consumo total de MS é igual ao somatório de ambos.

No submodelo variação do peso dos animais, é fundamental estabelecer as exigências do animal no dia simulado. A integração entre a variação do peso corporal, a qualidade da dieta ingerida e o custo energético do pastejo são utilizados para estabelecer as exigências nutricionais reais.

A exigência de mantença não é constante e depende do consumo de energia metabolizável (EM) da dieta e do peso metabólico do animal modificado pelo ajuste no tamanho de alguns órgãos (Dimarco et al., 2007). Realizou-se uma correção nos valores propostos pelo NRC (2000) e em concordância aos propostos por Tedeschi et al. (2002) do custo energético de $77 \mathrm{kcal}$ por unidade de peso metabólico em jejum por $126 \mathrm{kcal}$, considerando que esses animais não estão em jejum (Castellaro et al., 2007).

$\mathrm{RM}=\left[126.11 /\left(1+2,00262 * \mathrm{e}^{0,036 * \mathrm{Cdi}}\right) * \mathrm{~W}^{0,75}\right] / \mathrm{km}$

A eficiência de utilização da energia metabolizável pode variar de acordo com a função (de mantença ou de ganho) e com a natureza do alimento oferecido, que determina sua concentração energética (CE). A equação proposta por Blaxter (1977) para ganho de peso é:

$\mathrm{Kp}=18,4 * \mathrm{CD}+3,0$

Ocampos (1998) propôs ajustar a eficiência de utilização da energia quando o animal está em mantença:

$\mathrm{Km}=(55+6.694 * \mathrm{CD}) / 100$ 
O custo energético do pastejo de bovinos é produto do efeito combinado do gasto de energia próprio das ações de caminhar e pastejar (Di Marco \& Aello, 2003).

A metodologia empregada nos cálculos do custo energético do pastejo se baseia no trabalho de Osuji (1974), que estabelece quando a massa de forragem diminui de 2.800 para $370 \mathrm{~kg} \mathrm{MS} / \mathrm{ha}$ e o tempo gasto em pastejo aumenta de 8,2 horas a 12,3 horas. Di Marco \& Aello (2003) concluíram que o custo de movimentar $1,0 \mathrm{~kg}$ de peso $(0,49$ $\mathrm{kcal} / \mathrm{km}$ ) é relativamente constante entre espécies, coincidindo com os trabalhos apresentados por Brody (1945). Neste sentido, Osuji (1974) estabeleceu um custo para o consumo de alimento de 0,62 e $0,45 \mathrm{kcal} / \mathrm{kg} / \mathrm{hora}$ para feno seco e pastejo, respectivamente. Di Marco \& Aello (2003), com base nos dados reportados por Brody (1945), estabeleceram que o custo energético de caminhar $1 \mathrm{~km}$ é de $0,5 \mathrm{kcal} / \mathrm{kg}$ de peso corporal. A partir desses dados, estabeleceu-se que o custo energético de pastejar uma hora e caminhar um quilômetro são similares. Assim, para efeito do modelo, foi empregado o valor de $0,49 \mathrm{kcal} / \mathrm{kg}$ de peso do animal por hora de pastejo, assumindo-se que, acima de $2.800 \mathrm{~kg}$ massa seca/ha, o tempo gasto estabiliza-se em 8 horas.

$$
\begin{aligned}
& \mathrm{HP}=12,92428+0,0016872428 * \mathrm{DD} \\
& \mathrm{CC}=\mathrm{HP} * 0,49 * \mathrm{PC}
\end{aligned}
$$

O consumo de energia metabolizável, as exigências de mantença e o custo energético de pastejo (CC) são determinados para que seja possível calcular o balanço de energia para ganho de peso do animal. A partir disso, o modelo emprega a fórmula de Allende (2003), baseada nas equações propostas por Gought (1975):

$$
\mathrm{GP}=\left(\mathrm{Bal}^{+} * \mathrm{Kp}\right) / 1,5+0,3 * \mathrm{EL} \mathrm{gp}^{*} \mathrm{Kp}+0,0045 * \mathrm{PC}
$$

Supondo-se que o consumo de energia metabolizável é menor que as exigências de mantença do animal somadas ao custo energético de pastejo, haverá perda de peso, o ganho de peso terá valor negativo modificando a equação anterior:

$\mathrm{PerP}=\left(\mathrm{Bal}^{-*} \mathrm{Km}\right) / 1,5+0,3 * \mathrm{EL} \mathrm{gp}^{*} \mathrm{Km}+0,0045 * \mathrm{PC}$

No modelo pastagem, os resultados da taxa de acúmulo (kg MS/ha/dia) são incluídos na base de dados e considerados como o crescimento potencial das pastagens avaliadas. Esse valor é afetado por diferentes fatores, como precipitação, temperatura, fertilidade e compactação do solo, além da disponibilidade instantânea. Assim, o emprego de taxas de acúmulo (kg/MS/ha/dia) incluídas na base de dados do modelo inclui, por si só, todos os fatores que poderiam afetá-la como restrição do modelo.

O modelo faz duas correções à taxa de acúmulo que determina o crescimento real da forrageira. A primeira é um fator de correção pela massa de forragem, a qual é calculada diariamente de acordo com a massa de forragem instantânea, delimitando taxa de crescimento máximo na faixa de 1.000 a $3.000 \mathrm{~kg}$ de $\mathrm{MS} / \mathrm{ha}$. Fora dessa faixa, o fator é modificado como função quadrática proposta por Brito et al. (1998). O segundo fator é produto da senescência e responde de forma linear à massa de forragem instantânea, assumindo valor 0,5\% para Brachiaria sp. (Lascano \& Estrada, 1987). FCDisp $=-0,531515+0,000697 *$ Df $-0,00000015 * \mathrm{df}^{2}$

Sen $=0,005 *$ Df

No submodelo de massa de forragem, a massa de forragem variou conforme a carga animal, o consumo, o período do ano, o tempo de ocupação e o período de descanso (neste modelo os dois últimos fatores são fixos).

O modelo faz um cálculo diário, que é tomado como média mensal considerando a massa de forragem anterior, a taxa de acúmulo proposta para o mês simulado, a senescência e o consumo voluntário em relação à taxa de lotação empregada. Quando é empregado feno, o modelo assume eficiência de aproveitamento de $75 \%$, que é descontada do consumo diário dos animais de modo a diminuir a pressão de pastejo.

DisFin $=($ Df $*$ FCDisp $*$ Sem $)-($ CVOF - CF*0.75 $)$

$\mathrm{Na}$ análise estatística para estimar a acurácia do modelo, foram empregados os ganhos diários de peso médios (GdP), que foram comparados aos valores observados em artigos, utilizados para validar o modelo.

A análise de regressão simples, um dos testes mais amplamente utilizados até hoje, é realizada utilizando-se médias de saídas do modelo e médias do sistema real, como observações pareadas. Além disso, regressões lineares foram traçadas entre valores preditos e observados, incluindo a análise dos efeitos residuais. Os resíduos foram calculados pela diferença entre os ganhos diários de peso (GdP) preditos pelo modelo e os observados.

A validação do modelo foi feita utilizando-se dados experimentais e os procedimentos estatísticos descritos por Rayburn \& Fox (1990), além das comparações dos modelos usados pelo CNCPS (Fox et al., 2003). Posteriormente, compararam-se os resultados de desempenho preditos pelo modelo e os resultados de desempenho observados para verificar a acurácia do modelo.

Uma revisão bibliográfica foi realizada nas publicações da Revista Brasileira de Zootecnia dos últimos cinco anos em busca de resultados de experimentos em pastejo que permitissem a validação do modelo, dos quais foram escolhidos quatro. Inicialmente, validou-se o consumo de matéria seca.

Para seleção dos artigos, utilizaram-se os seguintes critérios: ser um experimento em pastejo; conter o valor de massa de forragem no início do pastejo e a digestibilidade 
da matéria seca ou NDT da forrageira e do suplemento fornecido, os pesos de entrada e saída individuais dos animais ou as médias do lote ou ganho de peso médio por tratamento; que não sejam empregados aditivos (ex: monensina sódica) no processo; que as forrageiras empregadas fossem do gênero Panicum ou Brachiarias; taxas de lotação; dados oriundos de universidades ou centros de pesquisas com protocolos claramente definidos e tempo de pastejo por período definido.

Validações parciais foram feitas para o tamanho corporal, que não pôde ser obtido no mesmo trabalho, assim como todas as variáveis necessárias. Assim, empregaram-se dados médios de cada raça disponibilizados pelas Associações de criadores.
Foram selecionadas duas forrageiras mais representativas das regiões tropicais e subtropicais sul-americanas, Panicum sp e Brachiaria sp., e avaliada a quantidade de suplementação na época seca. A suplementação foi feita à razão de $1 \%$ de peso vivo e os dados (Tabelas 1 e 2) foram utilizados para avaliar o consumo de matéria seca como \% do peso corporal e os dados (Tabelas 3 e 4 ) para avaliar o ganho de peso médio.

O modelo apresenta algumas restrições, por incorporar pequena aleatorização na função de consumo, simulando variações individuais baseadas nas variações normais de consumo dos animais em pastejo. Nesse sentido, é possível a obtenção de resultados com algumas variações em simulações em condições similares, aproximando-se assim

Tabela 1 - Consumo de matéria seca em capim-tanzânia (Gontijo Neto et al., 2006)

\begin{tabular}{|c|c|c|c|}
\hline $\begin{array}{c}\text { Massa de forragem/pré-pastejo } \\
\text { (kg MS/ha) }\end{array}$ & DIVMS (\%) & Peso vivo inicial (kg) & $\begin{array}{l}\text { Consumo de MS } \\
(\% \text { peso vivo) }\end{array}$ \\
\hline 2.190 & 44,5 & 229,0 & 1,43 \\
\hline 2.830 & 49,6 & 229,0 & 2,00 \\
\hline 2.830 & 49,6 & 249,5 & 2,00 \\
\hline 3.737 & 52,8 & 229,0 & 2,40 \\
\hline 4.272 & 54,3 & 229,0 & 2,44 \\
\hline 4.272 & 54,3 & 249,5 & 2,44 \\
\hline
\end{tabular}

Tabela 2 - Consumo de matéria seca em capim-braquiária por novilhos com peso inicial de $138 \mathrm{~kg}$ (De Almeida et al., 2003)

\begin{tabular}{ccccc}
\hline $\begin{array}{l}\text { Massa de forragem/pré-pastejo } \\
\text { (kg.MS/ha) }\end{array}$ & DIVMS (\%) & Período do ano & $\begin{array}{c}\text { Consumo de matéria } \\
\text { seca (\% PV) }\end{array}$ & Espécie forrageira \\
\hline 3.057 & 54,8 & Seca (julho) & 2,5 & B. brizantha \\
3.057 & 47,4 & Seca (julho) & 2,8 & B. decumbens \\
3.485 & 45,6 & Seca (outubro) & 3,0 & B. brizantha \\
3.485 & 49,8 & Seca (outubro) & 2,7 & B. decumbens \\
3.521 & 50,5 & Águas (janeiro) & 2,5 & B. brizantha \\
3.521 & 48,1 & Águas (janeiro) & 2,5 & B. brizantha \\
3.481 & 49,6 & Águas (abril) & 2,5 & B. decumbens \\
3.481 & 48,2 & Águas (abril) & 2,1 & B. decumbens \\
\hline
\end{tabular}

Tabela 3 - Ganho diário de peso em capim-braquiária (Euclides et al., 2001)

\begin{tabular}{|c|c|c|c|c|c|}
\hline $\begin{array}{c}\text { Massa de forragem/pré-pastejo } \\
\qquad(\mathrm{kg} \mathrm{MS} / \mathrm{ha})\end{array}$ & DIVMS (\%) & Período do ano/Suplementação & $\begin{array}{l}\text { Peso vivo } \\
\text { inicial }\end{array}$ & $\begin{array}{c}\text { Ganho diário } \\
\text { de peso }\end{array}$ & $\begin{array}{l}\text { Dias } \\
\text { simulados }\end{array}$ \\
\hline 2.860 & 52,5 & Seco / Não & 197 & 170 & 120 \\
\hline 2.210 & 49,0 & Seco / Não & 305 & -130 & 120 \\
\hline 2.210 & 49,0 & Seco / Não & 317 & -320 & 120 \\
\hline 2.860 & 52,5 & Seco / Sim & 197 & 570 & 90 \\
\hline 2.210 & 49,0 & Seco / Sim & 305 & 640 & 90 \\
\hline 2.210 & 49,0 & Seco / Sim & 353 & 560 & 90 \\
\hline
\end{tabular}

Tabela 4 - Ganho diário de peso em capim-massai/capim-mombaça (Euclides et al., 2008)

\begin{tabular}{|c|c|c|c|c|c|c|}
\hline \multicolumn{2}{|c|}{ Massa de forragem/pré-pastejo } & \multirow{2}{*}{$\begin{array}{c}\text { DIVMS }(\%) \\
58,7\end{array}$} & \multirow{2}{*}{$\begin{array}{c}\text { Espécie forrageira } \\
\text { Capim-massai }\end{array}$} & \multirow{2}{*}{$\frac{\text { Período }}{\text { Águas }}$} & \multirow{2}{*}{$\begin{array}{c}\text { Ganho diário de peso } \\
425\end{array}$} & \multirow{2}{*}{$\frac{\text { Dias }}{180}$} \\
\hline (kg.MS/ha) & 2.839 & & & & & \\
\hline & 2.755 & 52,3 & Capim-massai & Seca & -16 & 90 \\
\hline & 1.959 & 62,8 & Capim-mombaça & Águas & 561 & 180 \\
\hline & 1.833 & 58,1 & Capim-mombaça & Seca & 113 & 90 \\
\hline
\end{tabular}


da realidade das pesquisas de campo. Não são incorporados diretamente os efeitos do ambiente sobre os animais.

O valor nutritivo das pastagens, expresso em concentração de NDT/kg, restringe-se a valores normais de forragem com nível de energia de 0,40 a $0,65 \mathrm{~kg} \mathrm{NDT} / \mathrm{kg}$ matéria seca.

A maioria das funções empregadas não foi desenvolvida com gado Bos indicus. Portanto, o modelo pode ser melhorado a partir da inclusão de correções nos algoritmos matemáticos empregados com dados obtidos de pesquisas realizadas nestas condições.

\section{Resultados e Discussão}

Na comparação do consumo de matéria seca observado e predito e na avaliação da dispersão dos pontos em relação a uma regressão perfeita $(\mathrm{Y}=\mathrm{X})$, o modelo tendeu a superestimar o consumo em condições de massa seca de forragem inferior a 2.000 e superior a $1.000 \mathrm{~kg} / \mathrm{ha}$. Com DIVMS inferiores a $45 \%$, o modelo apresenta consumo de MS superior ao observado. Neste trabalho, o consumo de MS aumentou de acordo com a massa seca de forragem e a qualidade da forrageira, mas os aumentos simulados não foram tão altos (15\% inferiores em média) quanto os observados. A equação estimativa do modelo proposto apresentou $\mathrm{R}^{2}$ de $63,95 \%$ para a primeira validação, enquanto a segunda validação apresentou $\mathrm{R}^{2}$ de $58,9 \%$ e foi inferior, com tendências opostas (Figura 2).

Os cálculos do consumo potencial de MS no modelo são corrigidos primeiro por massa seca de forragem e logo depois por digestibilidade da matéria seca da forragem, fato pelo qual o consumo simulado responde positivamente aos aumentos, tanto da digestibilidade como da massa de forragem presente. Os consumos de MS observados nos trabalhos empregados para validação não refletem forte associação entre digestibilidade e a massa de forragem, pelo qual a exatidão em geral foi prejudicada, pois, para o modelo, a associação é mais rígida.

Existe grande dificuldade de isolar os efeitos das características morfológicas e da estrutura das forrageiras tropicais sobre o consumo e comportamento animal. Em bovinos pastejando forrageiras tropicais, mesmo quando grandes quantidades de forragem estão disponíveis, existe dificuldade para preencher suas exigências nutricionais (Stobbs, 1970).

Gontijo Neto et al. (2006) estabeleceram altas correlações entre o consumo de matéria seca de animais em pastejo com a altura do dossel, a matéria seca total, a matéria verde seca disponível e a relação folha/colmo da forragem, determinando coeficientes de correlação de 0,$83 ; 0,90 ; 0,91$ e 0,88, respectivamente. Além desses elementos, têm-se outros relacionados às taxas de crescimento da forragem, como o índice de área foliar e a estrutura do dossel determinados pela morfologia da planta, os quais obviamente são importantes na determinação do consumo animal.

Existe grande carência de estudos com avaliação do consumo e desempenho animal em pastejo com descrição detalhada das condições da pastagem, quantidade, distribuição, estrutura e qualidade, que possibilitem compreender e interpretar corretamente os resultados, e também compará-los aos obtidos em outras pesquisas. Todo sistema real pode ser representado em mais de um modelo. Desse modo, a decisão de inserir variáveis representativas num modelo depende, principalmente, do

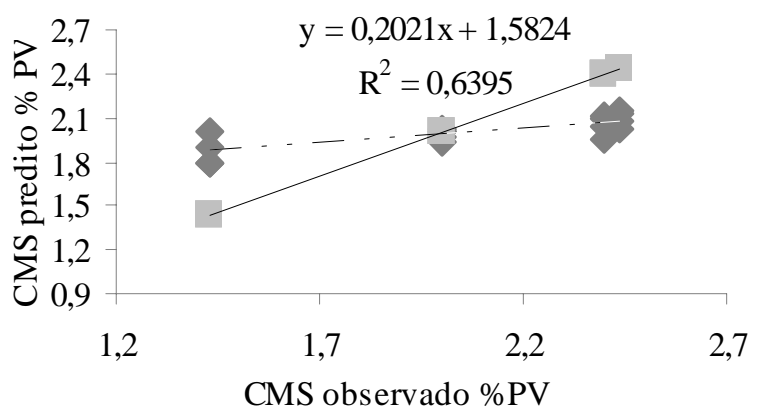

A linha cheia corres ponde a $\mathrm{Y}=\mathrm{X}$ e a linha tracejada corresponde à relação de mínimos quadrados (Validação 1)

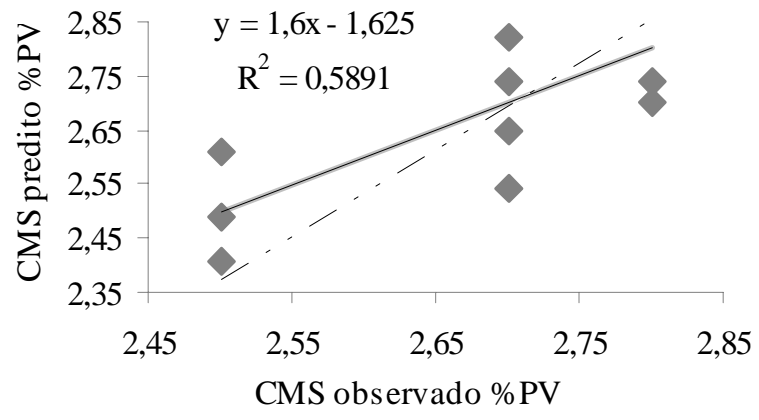

A linha cheia corresponde a $\mathrm{Y}=\mathrm{X}$ e a linha tracejada corres ponde à relação de mínimos quadrados (Validação 2)

Figura 2 - Relações entre os consumos de matéria seca observados para os dados experimentais nas validações 1 e 2. 
objetivo do teste e da capacidade para distinguir relações relevantes quanto aos objetivos propostos, pois a essência da modelagem sistêmica é a simplificação.

O consumo calculado pelo modelo foi ora superestimado ora subestimado, provavelmente em decorrência da nãoinclusão dos elementos discutidos anteriormente (altura, índice de área foliar, relação folha/caule etc.), os quais tem sido relevantes para explicar essa variável, embora de difícil tratamento, em virtude da carência de dados e da complexidade de sua inclusão.

Para a validação 3 da equação de predição de ganho de peso diário em jejum (GdP, g/animal/dia) com animais consumindo Brachiaria decumbens, o modelo apresentou predição com $R^{2}$ de $58,93 \%$ e, para a validação 4 de predição de ganho de peso diário em jejum com animais consumido capim-massai ou mombaça, o modelo apresentou $\mathrm{R}^{2} \mathrm{de}$ $55,48 \%$ (Figura 3). As melhores predições são obtidas com coeficientes de digestibilidade da matéria seca superiores aos 50\%. Houve tendência de superestimar o ganho de peso diário quando as digestibilidades da matéria seca foram baixas, em torno de 45\%; o mesmo comportamento foi observado para a equação de consumo (Figura 2). Validações para as estimativas de ganho de peso foram feitas por Lanna, Boin \& Fox (1994) com resultados experimentais de tourinhos Nelore. Esses autores obtiveram $\mathrm{R}^{2}$ de 57 e $51 \%$ para os modelos do CNCPS e NRC (1984), respectivamente, e estas validações foram feitas para condições de confinamento. Os valores comprovam que o modelo proposto neste estudo apresenta precisão quando empregados dados de digestibilidade e disponibilidade de matéria seca para predizer consumo e GdP. Não foi feita correção do ganho de peso por consumo observado, uma vez que o modelo não foi desenvolvido para isso. Um ponto importante para determinar GdP em pastejo é a exatidão na determinação do consumo.

As equações tenderam a superestimar ou subestimar tanto o consumo de matéria seca como o ganho de peso diário, especialmente quando foram baixos (Perdas de peso maiores do que $50 \mathrm{~g} / \mathrm{animal} / \mathrm{dia})$. O consumo foi superestimado em $20 \%$ acima do observado em consumos associados à pastagem com DIVMS inferior a $45 \%$. Em experimentos em pastejo, é comum observar consumos e ganhos de peso muito baixos (-100 a $0 \mathrm{~g} / \mathrm{animal} / \mathrm{dia})$, os quais nem sempre são explicados pela qualidade da dieta consumida e provavelmente são associados à presença de parasitas ou problemas de manejo decorrentes da metodologia.

Em geral o consumo potencial é definido como a capacidade máxima de ingestão do animal e está controlado por suas características físicas, desde que as características da dieta não sejam limitantes. Geralmente as equações para estimar o consumo potencial em kg não consideram o efeito da maturidade fisiológica do animal. Com base nesta premissa, optou-se pela estimativa proposta por CSIRO (1990), que considera o grau de maturidade por uma correção que avalia a relação do peso atual com o peso adulto e a idade do animal. Desta forma permitiria ao modelo avaliar ganhos compensatórios por meio da simulação de aumentos de consumo.

No caso das validações, todos os animais em cada processo de simulação tiveram a mesma idade. O modelo trabalha sobre uma média biológica dos animais submetidos ao tratamento. Portanto, pressupõe-se que exista uma

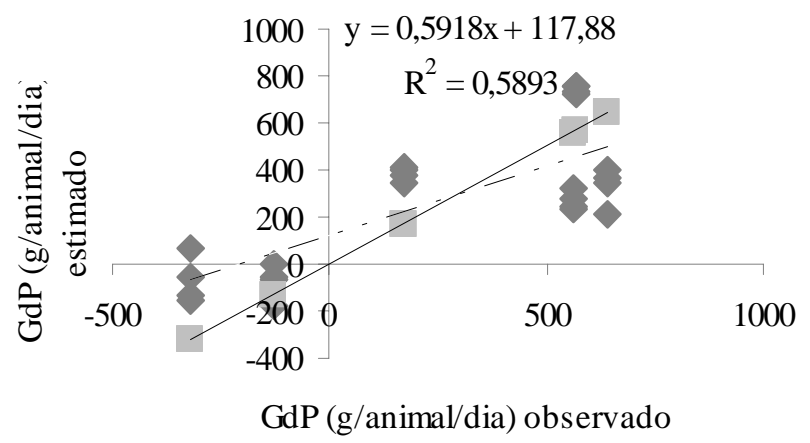

A linha cheia corresponde a $\mathrm{Y}=\mathrm{X}$ e a linha tracejada corresponde à relação de mínimos quadrados (Validação 3)

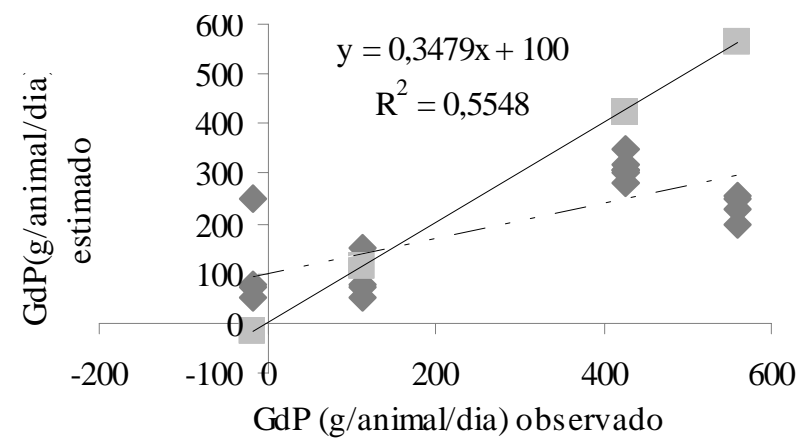

A linha cheia corresponde a $\mathrm{Y}=\mathrm{X}$ e a linha tracejada corres ponde a relação de mínimos quadrados (Validação 4)

Figura 3 - Relações entre os ganho de peso diários observados para os dados experimentais nas validações 3 e 4. 
tendência em superestimar resultados ruins e subestimar aqueles com limites maiores em decorrência da suposição da normalidade da distribuição da curva de ganhos de peso dos animais submetidos ao mesmo manejo.

\section{Conclusões}

O desenvolvimento do modelo possibilita maior compreensão do sistema pastoril e permite simular consumos, ganhos de peso e variações na massa de forragem, com o intuito de estabelecer parâmetros decorrentes da incidência da massa de forragem e da digestibilidade sobre o desempenho animal. $\mathrm{O}$ modelo pode ser considerado compatível para estimar o desempenho de bovinos, apesar das dificuldades para determinação do consumo animal. Portanto, é fundamental o desenvolvimento de equações de consumo em pastagens tropicais e de maior quantidade de estudos que ampliem o banco de dados existente. Além disso, é fundamental a padronização das metodologias de determinação da altura e de outras características de forrageiras tropicais para possibilitar sua inclusão em modelos de simulações posteriores.

\section{Referências}

AGRICULTURAL AND FOOD RESEARCH COUNCIL - AFRC. Feeding standars for Australian Livestock. Ruminant. Victoria: CSIRO, 1990. 266p.

AGUILAR, C. Simulación de sistemas. Aplicaciones em producción animal. 2.ed. Santiago: Facultad de Agronomia. Pontifícia Universidad Católica de Chile, 2007. 271p.

ALLENDE, R. Engorda de novillos en confinamiento. Evaluación mediante un modelo de simulación. 2003. 101f. Dissertação (Magister en Ciência Animal) - Facultad de Agronomia. Pontifícia Universidad Católica de Chile, Santiago.

BARIONI, L.G.; VELOSO, R.F.; MARTHA JUNIOR, G.B. Modelos para estimativa de desempenho de bovinos de corte e ovinos em pastagens. In: SIMPÓSIO INTERNACIONAL DE PRODUÇÃO ANIMAL: MODELOS PARA A TOMADA DE DECISÕES NA PRODUÇÃO DE BOVINOS E OVINOS, 2002, Santa Maria. Anais... Santa Maria: UFSM/Embrapa Pecuária Sul, 2002. p.5-60.

BLAXTER, K.L. Nutrition and the climatic environment. Boston: Butterworths, 1977. 314p.

BOVAL, M.; ARCHIMEDE, H.; CRUZ, P. et al. Intake and digestibility measured for heifers grazing tropical native grass at two stages of maturation (14 vs 28 days). Animal feed Science and Technology, v.134, n.1-2, p.18-31, 2007

BRITO, E.; AGUILAR, C.; CANAS, R. et al. Modelo de simulación para evaluar la sustenibilidade de las pasturas de la altillanura colombiana. I. Desarrollo y validación del modelo. Archivos Latinoamericanos de Produção Animal, v.6, n.1, p.1-38, 1998.

BRODY S. Bioenergetics and growth. New York: USA Reinhold Publishing Corporation, 1945. 1023p.

CARVALHO, P.F.; KOZLOSKI, G.; NUNES RIVEIRO FILHO, H. et al. Avanços metodológicos na determinação do consumo de ruminantes em pastejo. Revista Brasileira de Zootecnia, v.36, p.151-170, 2007 (supl. especial).
CASTEllaro, G.; KLEE, G.; CHAVARRIA, J. Um modelo de simulacion de sistemas de engorda de bovinos a pastoreo. Agricultura Técnica, v.67, n.2, p.163-172, 2007.

COMMONWEALTH SCIENTIFIC AND INDUSTRIAL RESEARCH ORGANIZATION - CSIRO. Feeding standarts for Australian Livestock ruminants. Melbourne, 1990. 266p.

DE ALMEIDA, G.R.; EUCLIDES, V.P.B.; NASCIMENTO, J.D. et al. Consumo, composição botânica e valor nutritivo da dieta de bovinos em pastos tropicais consorciados sob três taxas de lotação. Revista Brasileira de Zootecnia, v.32, n. 1, p.29-35, 2003.

DI MARCO, O.; AELLO, M. Costo energético de la actividad de vacunos em pastoreo y su efecto en la Produccion. Unidad Integrada Balcarse, 2003. Disponível em: <http:// www.inta.gov.ar/balcarce/info/documentos/ganaderia/bovinos/ nutrición/costo_eneg.htm>. Acesso em: 25/7/2006.

DI MARCO, O.; BARCELLOS, J.O.J.; DA COSTA, E. Crescimento de bovinos de corte. Porto Alegre: Departamento de Zootecnia/ Universidade Federal do Rio Grande do Sul/NESPRO, 2007. $278 \mathrm{p}$.

EUCLIDES, V.P.B.; EUCLIDES FILHO, K.; COSTA, F.C. et al. Desempenho de novilhos F1 Angus-Nelore em pastagens de Brachiaria decumbens submetidos a diferentes regimes alimentares. Revista Brasileira de Zootecnia, v.30, n.2, p. 470-481, 2001.

EUCLIDES. V.P.B.; MACEDO, M.C.M.; ZIMMER. et al. Avaliação dos capins mombaça e massai sob pastejo. Revista Brasileira de Zootecnia, v.37, n.1, p.18-26, 2008.

FOX, D.G.; TYLUTKI, T.P.; TEDESCHI, L.O. et al. CNCPS v. 5.0. The net carbohydrate and protein system for evaluation herd nutrition and nutrient excretion, model documentation. Ithaca: Cornell University; Animal Science Department, 2003 (Mimeo, 21).

GONTIJO NETO, M.M.; EUCLIDES, V.P.B.; NASCIMENTO JR., D. et al. Consumo e tempo diário de pastejo por novilhos nelore em pastagem de capim Tanzânia sob diferentes ofertas de forragem. Revista Brasileira de Zootecnia, v.35, n.1, p.6066,2006

GOUGHT, H.C. Energy allowance and feeding systems for ruminants. London: Her Majesty Stationery Office Government Bookshops, 1975. 79p.

HOLMES, W.; JONES, J. [1964]. The efficiency of utilization of fres grass. Proceeding of the Nutrition Society, v.23, p.88- 89. Disponível em: <http://journals.cambridge.org/action/ displayFulltext type $=1 \&$ fid $=784468 \&$ jid $=$ PNS \& volume $I d=23$ \&issueId=01\&aid=784460>. Acesso em: 27/8/2007.

INSTITUTE NATIONAL DE LA RECHERHCE AGRONOMIQUE - INRA. Ruminant nutrition. Paris: John Libbey Eurotext, 1989. 389p.

LANNA, D.P.D.; BOIN, C.; FOX, D.G. Validação do CNCPS e do NRC (1984) para estimativa dos requerimentos nutricionais e desempenho de zebuínos em crescimento. In: REUNIÃO ANUAL DA SOCIEDADE BRASILEIRA DE ZOOTECNIA, 1994, Maringá. Anais... Maringá: Sociedade Brasileira de Zootecnia, 1994. p. 480.

LASCANO, C.; ESTRADA J. Informe anual. Programa de pastos tropicales. Antioquia: CIAT, 1987. 62p.

LIMA, M.L.P.; BERCHIELLI, T.; NOGUEIRA, J.R. et al. Estimativa do consumo voluntário do capim-Tanzania (Panicum maximun, Jacq. c.v. Tanzânia) por vacas em lactação sob pastejo rotacionado. Revista Brasileira de Zootecnia, v.30, n.6, p.1919-1924, 2001.

NATIONAL RESEARCH COUNCIL - NRC. Nutrients requirement of beef cattle. Washington, D.C.: National Academy Press, 2000. $242 p$.

OCAMPOS, D. Produccion de leche em el subtropico paraguayo com vacas producto de cruzamientos Holando * Cebu Modelo de Simulacion. 1998. 187f. Dissertação (Mestrado em Produção Animal) - Curso de Pós-graduação em Produção Animal, Pontificia Universidad Católica de Chile. 
OSUJI, P. The physiology of eating and the energy expenditure of the ruminant at pasture. Journal of Range management, v.27, n.6, p.437-443, 1974.

RAYBURN, E.B.; FOX, D.G Predicting growth and performance of Holstein stress. Journal of Animal Science, v.68, p.788-798, 1990.
STOBBS, T.H. Automatic measurement of grazing time by dairy cows on tropical grass and legume pastures. Tropical Grasslands, v.4, n3, p.237-244, 1970.

TEDESCHI, L.O; BOIN, C; FOX, D.G. Energy requirements for maintenance and growth of Nellore bulls and steers fed high-forage diets. Journal of Animal Science, v.80, n.6, p.1671-1682, 2002. 